\title{
Ueber eine entoptische Erscheinung bei Bewegung des Augapfels.
}

\author{
Von \\ Dr. Ernst Fuchs, \\ Professor der Augenhoilkwnde an der Universität zu Libtiłh.
}

\section{Hierzu Tafel I.}

Ich beobachte an meinen Augen eine entoptische Wahrnehmung, welche sich anf Veränderungen im gelben Fleck, gesetzt durch mechanisehe Gewalt, zurückführen lässt. Dasselbe Phänomen wurde schon in ähnlicher Weise von Purkinje gesehen und besehrieben; doch gieng es hier ebenso wie mit vielen anderen der zahlreichen entoptischen Erscheinungen, welche Purkinje entdeckt hatte: keiner der späteren Autoren war im Stande, sich dieses Phänomen zur Ansehauung zu bringen und dessen Existenz zu bestätigen. Dies veranlasste mich schon vor einigen Jahren, die Erscheinung genauer zu studiren und die erhaltenen Resultate niederzuschreiben. Ich glaubte dies umsomehr thun zu sollen, als ich das Phänomen in etwas anderer Weise als Purkinje sah, und weil ich eine ron Purkinje abweichende und, wie mir seheint, stichhaltigere Erklärung dafür gefunden habe.

Um die erwähnte entoptische Erscheinung zu beobachten, bringe ich an die rechte Seite meines Kopfes eine v. Graefe's Archiv fur Ophthalmoiogie, XXVII. 3. 
gleichmässig weisse, hell beleuchtete Fläche. Ich setze mich z. B. so gegen eine weissgetünchte Wand, dass ich dieselbe zur rechten Seite habe, oder ich halte mit der Hand einen Bogen weissen Papieres nach reehts von meinem Kopfe. Die Distanz der weissen Fläche vom Auge ist gleichgültig; man kann das Papier sogar ganz nahe ans Auge bringen, indem man es an die Wange anlegt. Nun wende ich die Augen, welche bis jetzt gerade nach vorne gerichtet waren, rasch, mit einem gewaltsamen Rucke, nach reehts auf die weisse Fläche und erblicke dann auf derselben mit dem rechten Auge folgende Lichtfigur.

Ich sehe zunächst einen grossen farbigen Ring, der jedoch in der Regel sehr unvollständig und undeutlich ist. Auf seiner nasalen Seite bemerke ioh, in den Ring eingetragen, zwei Systeme von übereinander geschichteten concentrischen krummen Linien. Die Fig. 1 giebt die Frscheinung wieder, so wie ich sie auf $50 \mathrm{~cm}$ Entfernung projicirt sehe, wobei sie also verhältnissmässig gross erscheint. Die Linien sind äusserst zart und nicht scharf contourirt; es wäre eigentlich besser zu sagen, es handle sich um eine Aufeinanderfolge abwechselnd heller und dunkler, farbloser Streifen. Dieselben besitzen eine sehwach bogenförmige Krümmung, welche in der beigegebenen Zeichnung einem Radius von ungefähr $12 \mathrm{~cm}$ entspricht. Doch sind die Bogen nicht ganz regelmässig, indem sie zahłreiche kleine, wie wellenförmige Krümmungen aufweisen; auch sind sie nicht alle, gleich lang. Ein freier Zwischenraum a in der Mitte scheint sie in zwei isolirte Gruppen zu trennen. Die Zahl der Linien ist etwa 20.

Lange Zeit sah ich die Erscheinung nur in der geschilderten Weise. Eines Tages bemerkte ich zufallig, dass auch der anscheinend freie Zwischenraum nicht ganz leer sei. Es war dies der Fall, als ich schräge auf eine in der Sonne glitzernde Wasserfläche sah. Ich konnte mich überzengen, dass sich die Linien in regelmässiger Weise 
auch über den Zwischenraum fortsetzten and mit einander verbanden, $\mathrm{j} a$, dass $z$ wischen denselben noch weitere feine Linien zum Vorscheine kamen. Diese letzteren reichten jedoch nicht über die Grenzen des grossen Zwischenraumes hinans. Sie halbirten die Intervalle zwischen den längeren Linien, so dass hier die doppelte Zahl von Bogen vorhanden war. Alle zusammen waren im Bereiche des grossen Zwischenraumes ausserordentlich fein und blass, so dass ich sie bis dahin nicht wahrgenommen hatte. Auch jetzt noch sehe ich für gewöhnlich die Mitte der Figur vollkommen leer und nur bei sehr greller und blendender Beleuchtung werde ich die feinen Linien in der Mitte gewahr. Ungefähr in die Mitte des grossen Zwischenraumes fallt der Fixationspunkt.

Geübt durch lange Beobachtung dieses Phänomens sehe ich die Figur mit dem rechten Auge ausserordentlich leicht. Es genügt, mich rasch nach Jemandem umzusehen, damit ich dieselbe, auch ohne besonders darauf zu achten, wahrnehme. Andererseits wird die Beobachtung dadurch erschwert, dass die Figur nur für einen Moment sichtbar ist und zwar unmittelbar nach der gewaltsamen Bewegung des Anges nach aussen. Sobald das Auge in der stark abducirten Stellung zur Ruhe gekommen ist, verschwindet sofort die Erscheinung. Es ist deshalb nicht möglich, mit Genauigkeit die Anzahl der Linien zu zählen oder ihre Länge, die Breite der Intervalle u. s. w. zu messen. $\mathrm{Zu}$ annähernden Resultaten kam ich auf folgende Weise. Ich projicirte die Erscheinung auf ein in einer bestimmten Entfernung gehaltenes Blatt Papier, dann zeichnete ich sie darauf $a b$, sowie ich sie ungefähr gesehen hatte. Ich trachtete nun wiederholt, das Phänomen auf dasselbe Blatt Papier projicirt zu erhalten, so dass die Figur auf oder neben die Zeichnung fiel, welche ich dann immer wieder mit der Originalerseheinung verglieh und so lange darnach corrigirte, bis sie der Erschei- 
nung selbst so genau als möglich zu entsprechen schien. Indem ich diese Beobachtungen bei verschiedenen Entfernungen der Fläche, auf welche projicirt wurde, anstellte, erhielt ich folgende Mittelwerthe, welche nach dem reducirten schematischen Auge von Donders*) für die Distanzen auf der Netzhaut berechnet sind.

Länge der Figur (bb) . . . . . . . $=2,25 \mathrm{~mm}$

Breite derselben . . . . . . . . =0,94 "

Breite des grossen Zwischenraumes a . . = $=0,46$ "

Breite einesIntervalles zwischen den grossen

Linien . . . . . . . . $=0,064$,

Breite eines Intervalles zwischen den feinen

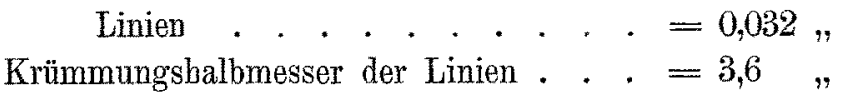

Die Lage der Liniensysteme im Raume wird durch Fig. 2 veranschaulicht. Beim Blicke nach rechts in der Horizontalebene stehen die Bogen senkrecht auf dem horizontalen Meridiane, ihre Concavität sieht nach aussen. Beim Blicke nach aussen oben stehen die Bogen schräg von aussen oben nach innen unten und wenden ihre Concavitat nach unten aussen; beim Blicke nach aussen unten nehmen sie umgekehrt eine von oben innen nach aussen unten gehende Richtung mit der Concavität nach aussen oben an.

Ich kann dieselbe Erscheinung auch bei geschlossenen Augen wahrnehmen, wenn nur noch etwas Licht durch die Lider selbst ins Auge dringt. Ich sehe dann den grossen hellen, etwas ovalen Ring mit dunkler Mitte besonders deutlich (Fig. 3). Sein scheinbarer Durchmesser ist etwa $3 \mathrm{~cm}$; die an seiner inneren Seite befindlichen Liniensysteme sind gut kenntlich, doch nicht so scharf und deutlich als wenn ich das Experiment mit offenen Augen

*) Die Anomalien der Refraction und Accommodation p. 151. 
anstelle. Wenn ich die geschlossenen Augen noch mit der Hand bedecke, so dass gar kein licht mehr in dieselben fällt, bin ich nicht mehr im Stande, etwas von der Lichtfigur wahrzunehmen. - Wenn ich die Augen rasch nach links drehe, sehe ich dasselbe Phänomen mit dem linken Auge auf der linken Seite, doch lange nicht so leicht und so dentlich, als dies bei der früheren Versuchsanordnung mit dem rechten Auge der Fall war.

Purkinje hat offenbar schon dieselbe Erscheinung gesehen. In seinen: „Beobachtungen and Versuchen zur Physiologie der Sinne"*) beschrieb er die Lichtfigur, welche er wahrnimmt, wenn er das wohl bedeckte Auge schnell und kräftig gegen den äusseren Augenwinkel dreht. Er sieht zunächst einen hellen Ring mit dunklerer Mitte; um den Ring herum ist der Grund lichter und zeigt an der gegen den Mittelpunkt des Sehfeldes gelegenen Seite feine mit dem Ring concentrische, wechselweise lichte and dunkle Streifen, die nach innen immer kürzer werden und hin and wieder unterbrochen sind. Fig, 4 ist eine Copie der Purkinje'schen Zeichnung. Man sieht daraus zugleich, worin die von Purkinje gesehene Lichtfigur von der von mir gegebenen Beschreibung abweicht.

Helmholtz**) sah bei rascher Auswärtswendung des Auges ebenfalls einen lichten Kreis, den er auf die Zerrung des Opticus zurückführt, welcher aber nicht dem leuchtenden Kreise entspricht, wie ihn Purkinje und ich sahen. Die an den Kreis sich anschliessenden Bogenlinien konnte er nicht walnrnehmen.

A ubert***) and Czermak $\dagger$ ) scheinen ebenfalls nur die Lichtfigur, nicht aber die Bogenlinien gesehen zu haben. Keiner meiner Collegen, denen ich den Versuch in geeig-

*) 1825, I. Bd. pag. 79 u. 80 und Fig. 22.

**) Physiologische Optik p. 198.

***) Physiologie der Netzhaut p. 338.

†) Wiener Akademieberichte, XV. Bd, p. 455. 
neter Weise anstellen liess, war im Stande, sich die Bogenlinien zur Anschauung zu bringen.

Purkinje erklärte die gesammte Erscheinung (Lichtring und Bogenfigur) damit, dass durch die Zerrung des Opticus ein elektrischer Gegensatz in der Substanz des Nerven erregt würde. Diese Erklärung ist, abgesehen von anderen Mängeln, auf keine Weise im Stande, über die Entstehung der Bogenlinien Auskunft zu geben.

Die plausibelste Erklärung des Phänomens scheint mir folgende zu sein: Bei sehr rascher Auswärtswendung des Auges wird der hintere $\mathrm{Pol}$ desselben vehement nach innen gedreht. Dies kann nicht ohne Zerrung des Opticus abgehen, welche sich in die unmittelbar umgebende-Netzhaut fortpflanzt. Die Erschütterung der letzteren giebt sich als Lichtring zu erkennen. In der Gegend des hinteren Poles, wo die Netzhaut besonders dünn ist, rommt es sogar zu feinen Faltungen der Netzhaut, welche entoptisch als bogenförmige linien wahrnehmbar werden.

Ich gehe nun daran, die einzelnen Punkte dieser Erklärung genauer zu begründen.

1) Zerrung des Opticus. Damit die genannte Erscheinung zu Stande kommen könne, muss die Wendung des Auges sehr rasch und kräftig geschehen. Ja dies allein ist nicht einmal hinreichend, sondern es muss noch ein besonderes Moment hinzukommen, welches die Zerrung unvermeidlich macht. Man sieht nämlich das Phänomen niemals, wenn man das Auge, sei es auch noch so kräftig, nach oben oder innen oder unten wendet*). Die Bewegung

*) Purkinje beschreibt (1. c. p. 138) einen Versuch, der darin besteht, die Ecke eines Kartenblattes in den inneren Augenwinkel zu bringen, dasselbe durch starke Einwärtsdrehung des Auges zu fixiren and dann mit einem stumpf zugespitzten Holze oder mit dem Knopfe einer Stecknadel einen Druck in der Gegend des hinteren Augenpoles anzubringen. Er sieht dann am Rande des Druckphosphens viele concentrische Streifen. Helmholtz 
muss gerade nach aussen, nach anssen oben oder aussen unten stattfinden. Dies weist darauf hin, dass hier die Musculi obliqui im Spiele seien, welche bei diesen Bewegungen des Auges thätig sind. Sie haben die Eigenthümlichkeit, dass ihre Insertionslinien im hinteren $\mathrm{Ab}$ schnitte des Bulbus liegen und also dem Opticus mehr oder weniger genähert sind. Dies gilt besonders rom Obliquus inferior, dessen Insertionslinie mit ihrem inneren Fnde dem hinteren Augenpole auf $2 \mathrm{~mm}$ nahe rückt.*)

Es wird also bei rascher Auswärtswendung des Bulbus, wobei nebst dem Rectus externus auch die beiden Obliqui thätig sind, der hintere $\mathrm{Pol}$ des Auges mit besonderer Heftigkeit nach innen gerissen; der Opticus muss diese Bewegung mitmachen, aber theils wegen des Trägheits momentes, theils weil das ihn umgebende Gewebe eine gewisse Zeit braucht, um ihm Platz zu machen, bleibt er ein wenig hinter der Bewegung des Bulbus zurück. Dies äussert sich darin, dass der intraoculäre Theil des Sehnerven vom inneren Rande des Foramen sclerae weggezerrt und gegen den äusseren Rand desselben angedrängt wird. Diese Ortsveränderung muss sich auch auf die den Opticus umgebenden Netzhauttheile fortpflanzen; dieselben werden an der inneren Seite des Sehnerven gedehnt, an der äusseren aber zusammengeschoben. Diese mechanische Veränderung bedingt das entoptische Phänomen des oben beschriebenen Lichtringes. Ich stelle mir vor, dass dèrselbe der Ausdruck einer den Opticus kreisförmig umgebenden Welle sei, welche an der ärsseren Seite des Opticus einer

und A ubert konnten diese Streifen nicht wahrnehmen. Ich sehe dieselben sehr deutlich und ungefähr ebenso wie sie Purkinje gezeichnet hat. Dieselben sind aber nicht mit denjenigen zu verwechseln, von welchen oben die Rede ist, schon deswegen nicht, weil sie stationär sind, d. h. so lange andanern, als man den Druck auf den Augapfel ausübt. Ferner sehe ich an ihnen auch keinerlei Unterbrechung in der Mitte.

*) Henle, Anatomie II. Bd. p. 694 . 
Verdichtung, an der inneren Seite einer Verdünnung (Zerrung) der Netzhaut entspricht. Es ist leicht, zu zeigen, dass diese Welle den Opticus zum Mittelpunkt hat und durch die Fovea centralis geht. Denn an letzterer Stelle, wo die Netzhaut sehr dünn ist, aussert sich die Verschiebung derselben nicht mehr als eine innigere Aneinanderpressung der einzelnen Netzhautelemente, sondern die Netzhaut legt sich in feine Falten, welche dem Rande der Papille concentrisch sind. Diese Falten werden entoptisch als jene Bogenlinien wahrgenommen, welche in den inneren Rand des Kreises eingetragen sind (Fig. 3). Ich werde später den Beweis erbringen, dass die Bogenlinien dem Fixationspunkte entsprechen; es geht somit auch der Lichtkreis an einer Stelle durch die Fovea centralis. Die Krümmungen der in den Kreis eingetragenen Bogenlinien entsprechen vollkommen der Krümmung des übrigen Lichtkreises; sie können als zu ihm gehörige Bogen betrachtet werden. Da wir nun später sehen werden, dass die Bogenlinien den Mittelpunkt der Papille zum Centrum haben, so folgt daraus anch, dass der ganze leuchtende Kreis die Opticusm scheibe als Mittelpunkt habe.

Warum geht jener Kreis, welcher auf der äusseren Seite der grössten Verdichtung der Netzhant entspricht, gerade durch den hinteren Pol? Man denke sich einen Querschnitt durch Papille und Netzhaut, welcher gerade durch die Fovea centralis geht. Wenn man nun die beiden Enden des Schnittes einander zu nähern versuchte, müsste dies zu einer Faltung an der dünnsten Stelle des Schnittes, also in der Fovea, führen. Das ist denn auch am lebenden Auge bei rascher Auswärtsbewegung in der That der Fall, nur dass hier die ganze äussere Halfte der Netzhaut in ähnlicher Weise zusammengeschoben wird. Die Falten der Fovea können sich wohl als solche nicht weit nach aufwärts und abwärts fortsetzen, weil die Netzhaut hierzu za dick ist, wohl aber finden wir als directe Fortsetzung der 
Falten einen Halbkreis, welcher der grössten Verdichtung der Netzhaut durch Aneinanderdrängung ihrer Elemente entspricht.

Helmholtz hat bei rascher Auswärtswendung des Blickes ebenfalls den farbigen Ring gesehen, welcher jedoch bei ihm dem Umfange der Sehnervenscheibe entspricht und der Ausdruck der unmittelbar am Rande des Sehnerven stattfindenden Zerrung ist.

2) Localisation der Bogenlinien in der Netzhaut. Die Lage der Linienfigur in der Netzhaut lässt sich leicht eruiren, wenn man auf das Blatt Papier, worauf man die Erscheinung projicirt, sich ein Fixationszeichen macht, am besten einen kleinen Buchstaben, um sicher zu sein, dass man wirklich central fixirt. Man wird dann finden, dass der Fixationspunkt ungefähr in die Mitte des grossen Zwischenraumes a fällt. Ich sehe nicht selten gleichzeitig mit diesem Phänomen auch die Purkinje'sche Aderfigur auftauchen and kann auch aus dieser die Lage der Fovea centralis entnehmen, welche immer mit dem. genannten $Z$ wischenraume zusammenfällt.

3) Die Bogenlinien sind der Ausdruck einer zarten Faltung in der Netzhaut. Gleich das erste Mal, als ich sie wahrnahm, bekam ich diesen Eindruck von ihnen. Ich glaube auch zu bemerken, dass die Gegenstände, welche ich durch die Bogenfigur sehe, leicht unregelmässig, wie gewellt, erscheinen. Dies ist namentlich der Fall, wenn ich als Projectionsebene eine mit kleinem Drucke bedeckte Fläche wähle. Auch die schnelle Vergänglichkeit des Phänomens spricht dafür, dass demselben Falten zu Grunde liegen, welche sich beim Stillstehen des Auges alsbald wieder ausgleichen. Krümmung und Richtung der Linien stimmt vollständig mit ihrer Deutung als Falten in der Macula lutea überein.

Es ist nach dem oben geschilderten Mechanismus der Zusammenschiebung der Netzhaut rorauszusetzen, dass 
die Falten, welche sich in der Gegend des hinteren Poles bilden, concentrisch mit dem äusseren Rande der Papille verlaufen. Nimmt man die Papille als vollkommen rund an, so würden die Falten Kreisbogen entsprechen, deren Centrum mit dem Centrum der Papille zusammenfällt. Diese Vermuthung wird durch. die Messung der Krümmung bestätigt. Ich habe den Krümmungsradius dieser Bogen mit 3,6 mm gefunden. Damit stimmt die Distanz zwischen Mittelpunkt der Fovea centralis und Mittelpunkt der Papille ziemlich genau uberein, welche nach Weber 3,8 , nach Krause 3,28 und $3,6 \mathrm{~mm}^{*}$ ) beträgt.

Die in der Netzhaut nasalwärts (gegen die Papille) gerichtete Concavität der Falten muss, nach aussen projicirt, nach der Schläfenseite sehen, wie es in der That der Fall ist. Beim Blicke nach aussen oben ist die Richtung der Bogen eine von aussen oben nach innen unten gehende, ihre Concavitait sieht nach aussen unten. Dies kommt daher, dass bei der genannten Blickrichtung in Folge Action des Obliquus inferior der verticale Meridian des Auges mit seimem oberen Ende nach aussen geneigt wird, also die Papille nach innen oben von der Fovea centralis zu stehen kommt.

Beim Blicke nach aussen unten macht sich in analoger Weise die Wirkung des Obliquus superior auf den verticalen Meridian geltend. Immer ist zu bedenken, dass die nach aussen auf eine Fläche projicirten Linien gerade die entgegengesetzte Lage einnehmen müssen, wie die Falten in der Netzhant.

4) Beziehung der Bogenlinien zum gelben Flecke und zur Netzhautgrube. Die Bogenfigur dürfte in ihrer Ausdehnung ungefähr der Macula lutea entsprechen. Die Breite der Figur beträgt nämlich $0,94 \mathrm{~mm}$.

*) Siehe Helmholtz 1. c. p. 22. 
Müller*) gibt den Durchmesser der centralen Partie des gelben Fleckes, welche stärker gefärbt und daher allein scharf abgrenzbar ist, mit 0,8-1,5, ein andermal mit $0,53-0,882 \mathrm{~mm}$ an. Die Ausdehnung der gelben Färbung ist also ziemlich variabel; auch hat sie mit der Faltenbildung in der Netzhaut direct nichts zo thun, wohl aber vielleicht der Umstand, welchen Müller**) erwähnt, dass er im gelben Flecke die bekannten dreieckig abgeschnittenen oder getheilten inneren Enden der Radialfasern der Netzhaut nicht finden konnte. Dies würde auf ein lockeres, der Faltenbildung günstiges Gefüge der Netzhaut im Bereiche der Macula lutea hinweisen.

Da die Länge der Bogenfigur 2,25 mm beträgt, so muss angenommen werden, dass die Falten nach oben und nach unten das Areal des gelben Fleckes übersehreiten.

So lange ich die Bogenlinien im Centrum vollständig unterbrochen sah, blieb mir die ganze Erscheinung unbegreiflich. Nachdem ich aber gefunden hatte, dass sich die Linien über den centralen Zwischenraum hin erstrecken, dass sie daselbst sogar in verdoppelter Anzahl, nur viel lichtschwächer vorhanden waren, glaubte ich folgende Erklärung dafür aufstellen zu können: Die Netzhaut haftet in der Forea cent. besonders fest an der Unterlage, indem sich hier die pigmentirten Fortsätze des Chorioidealepithels weiter als sonst zwischen die Zapfen vorschieben. ${ }^{* * *}$ ) Dieser Umstand gestattet nicht, dass die Falten, welche sich im Bereiche der Macula Iutea bilden, in gleicher Höhe über die Netzhautgrube hinwegsetzen. Die Falten mussten an dieser Stelle niedriger werden; da aber doch die Zusammenschiebung der Netzhaut an der Stelle der Netzhautgrube die gleiche ist, als unmittelbar

*) Gesammelte Schriften, heransg. von Becker. Leipz. 1872. pag. 108.

**) l. c. p. 24 und 99.

***) Müller l. c. p. 140 . 
darüber und darunter, konnte die Herabminderung der Faltenhöhe nur dadurch ausgeglichen werden, dass sich zwischen den primären Falten eine zweite Serie von Falten bildete, so dass nun die doppelte Anzahl von Falten vorhanden war, welche aber nur die Hälfte der Höhe zu besitzen brauchte. Die grosse Dünnheit der Netzhaut an der Forea cent. gestattete leicht diese feine Faltung der Membran, während doch andererseits die innige Adhärenz dieser Stelle an die Unterlage höhere Falten unmöglich gemacht hätte. Die Ausdehnung des grossen Zwischenraumes in der Bogenfigur stimmt in der That mit der Grösse der Netzhautgrube ubberein; seine Breite' beträgt $0,46 \mathrm{~mm}$, während Müller*) die Ausdehnung jener Netzhautstelle, welche der Faserschichte entbehrt, mit 0,4 bis $0,6 \mathrm{~mm}$ angibt.

5) Die Höhe der Falten kann selbstrerständlich nur als eine minimale gedacht werden. Einen Anhaltspunkt dafür gewährt die Breite des Intervalles zwischen je zwei Bogenlinien. Da die Breite der ganzen Figur $0,94 \mathrm{~mm}$ beträgt und etwa 18 Intervalle vorhanden sind, so ist die Breite eines jeden Intervalles $0,052 \mathrm{~mm}$. In der Mitte der Figur, wo die Zahl der Falten doppelt so gross ist, beträgt die Breite eines Intervalles nur $0,026 \mathrm{~mm}$. Nehmen wir nun der einfacheren Rechnung halber an, dass die Falten im Querschnitte Kreisbögen darstellten. a bis a, in Fig. 5 sei der schematisohe Querschnitt einer Falte. Selbst wenn man sich eine solche Falte sehr steil denkt, so würde ihre Hohe höchstens die Hälfte des Intervalles $=2 \mathrm{r}$ sein können, also in der Mitte der Linienfigur $0,013 \mathrm{~mm}$. Nun ist die Breite eines Zapfens in der Fovea centralis ungefähr $0,0025 \mathrm{~mm}^{* *}$ ); es kommen also auf eine ganze Falte, deren Basis aa, $0,026 \mathrm{~mm}$ ist, etwa

*) 1. c. p. 109 und 111.

**) Nach Müller (1. c. p. 139) 0,003, nach Max Schultze 0,002-0,0025 (siehe Schwalbe in Graefe-Saemisch I. Bd, p. 438). 
10 Zapfen und auf jede Hälfte der Falte (von ihrem tiefsten Punkte bis zum Gipfel $a_{1}$ ) 5 Zapfen. Auf diese 5 Zapfen, deren jeder eine Länge von durchschnittlich $0,075 \mathrm{~mm}$ besitzt*), vertheilt sich somit eine Niveandifferenz, welche im Maximum $0,013 \mathrm{~mm}$ betrăgt; das würde eine Verschiebung von 0,0026 des einzelnen Zapfens gegen seinen Nachbar geben, eine Verschiebung, welche ungefähr der Breite eines Zapfens gleichkommt. Diese Verschiebung ist an und für sich so klein, dass dieselbe ganz wohl vor sich gehen könnte, ohne dass dadurch eine nachtheilige Störung im Zusammenhange der Netzhaut gesetzt würde. Es hindert uns aber Niehts, die Höhe der Falten noch für viel geringer zu halten. Es genügt ja doch eine ganz minimale mechanische Reizung der. Netzhaut, um uns in der Form eines entoptischen Phänomens zum Bewusstsein zu kommen. Mir ist es sogar sehr wahrscheinlich, dass die Höhe der Falten eine viel geringere, etwa $1 / 2$ oder $1 / 4$ der angenommenen Grösse sei, u. z. aus folgendem Grunde: Man überlege, welches die totale Verkürzung der Netzhaut wäre für den oben angenommenen Fall, dass die Faltenhöhe die Hälfte der Faltenbasis beträgt. In diesem Falle ist die Verkürzung der Netzhaut im Bereiche jeder einzelnen Falte $2 \mathrm{r} \pi-4 \mathrm{r}$. In der Mitte der Bogenfigur, wo die Breite des Intervalles 0,026 ist, ist $\mathrm{r}=\frac{0,026}{4}$, daher die Verkürzung 0,0148 mm. Da 36 Falten gezählt wurden, resultirt eine Gesammtverkürzung von $0,534 \mathrm{~mm}^{* *}$ ). Dies wäre die Distanz, um welche sich bei raseher Auswärtswendung des Bulbus der Opticus innerhalb des Foramen opticum

*) Müller (l.c. p. 109) gibt ihre Länge in der Fovea centr. mit $0,05 \mathrm{~mm}$, Max Schultze (Strickers Gewebelehre p. 1023) mit im Maximum $0,1 \mathrm{~mm}$ an.

**) Dasselbe Resultat erhäl man natürich, wenn man die Berechnung für die seitlichen Theile der Figur mit den grösseren Intervallen anstellt. 
verschieben müsste. Diese Verschiebung ist nun entschieden zu gross, so dass man sich aus diesem Grunde die Höhe der Falten viel geringer denken muss.

Zum Schlusse sei noch Folgendes bemerkt: Wenn sich die Verschiebung der Netzhaut nach aussen durch den andrängenden Sehnerven erst in der Macula lutea durch Faltenbildung compensirt, so müssen diejenigen Netzhauttheile, welche zwischen dem äusseren Rande der Papille und dem inneren Rande der Macula lutea liegen, eine Verschiebung auf der Unterlage um die ganze Länge der Verkürzung erleiden. Man sollte daher denken, dass selbst in dem Falle, wo die gesammte Versehiebung nur $1 / 10$ eines Millimeters beträgt, sie hinreichend sein würde, damit die Stäbchen und Zapfen sämmtlich aus den Pigmentepithelzellen herausgerisssen, und so die äusseren Netzhautschichten zerstört würden. Diese Folgerung würde aber nur dann stichhaltig sein, wenn man sich vorstellte, dass die Verschiebung zwischen der Stäbchen- und Zapfenschichte einerseits and der Epithelschichte andererseits stattfände, dass die eine dieser Schichten auf der anderen gleite. Eine solche Vorstellung wird aber durch nichts gestützt; es ist vielmehr weit wahrscheinlicher, dass die Verschiebung auf die ganze Dicke der Netzhaut und auch der Aderhaut sich vertheile. Namentlich bei letzterer kann wegen des losen Zusammenhanges ihrer einzelnen Schichten untereinander und mit der unterliegenden Sclera sehr leicht eine ausgiebige Verschiebung der gesammten Membran angenommen werden. Indem sich so die Bewegung: auf die Netzhaut und Aderhaut vertheilt, ist sie an keinem Punkte so bedeutend, um eine dem anatomischen Zusammenhange der Elemente schädliche Höhe zu erreichen. 
D. Graefí's Archio Bet.XXVIT, 3.

Taf. 1

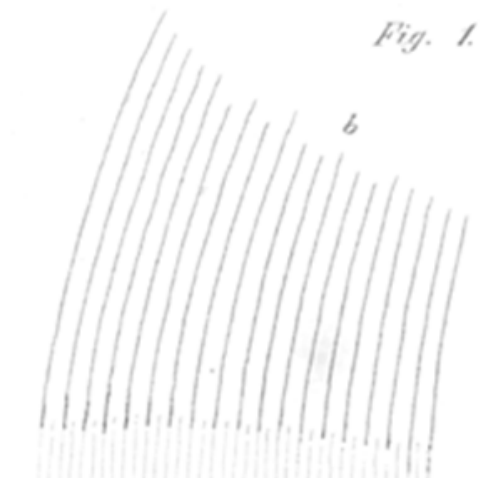

Fig. 2.
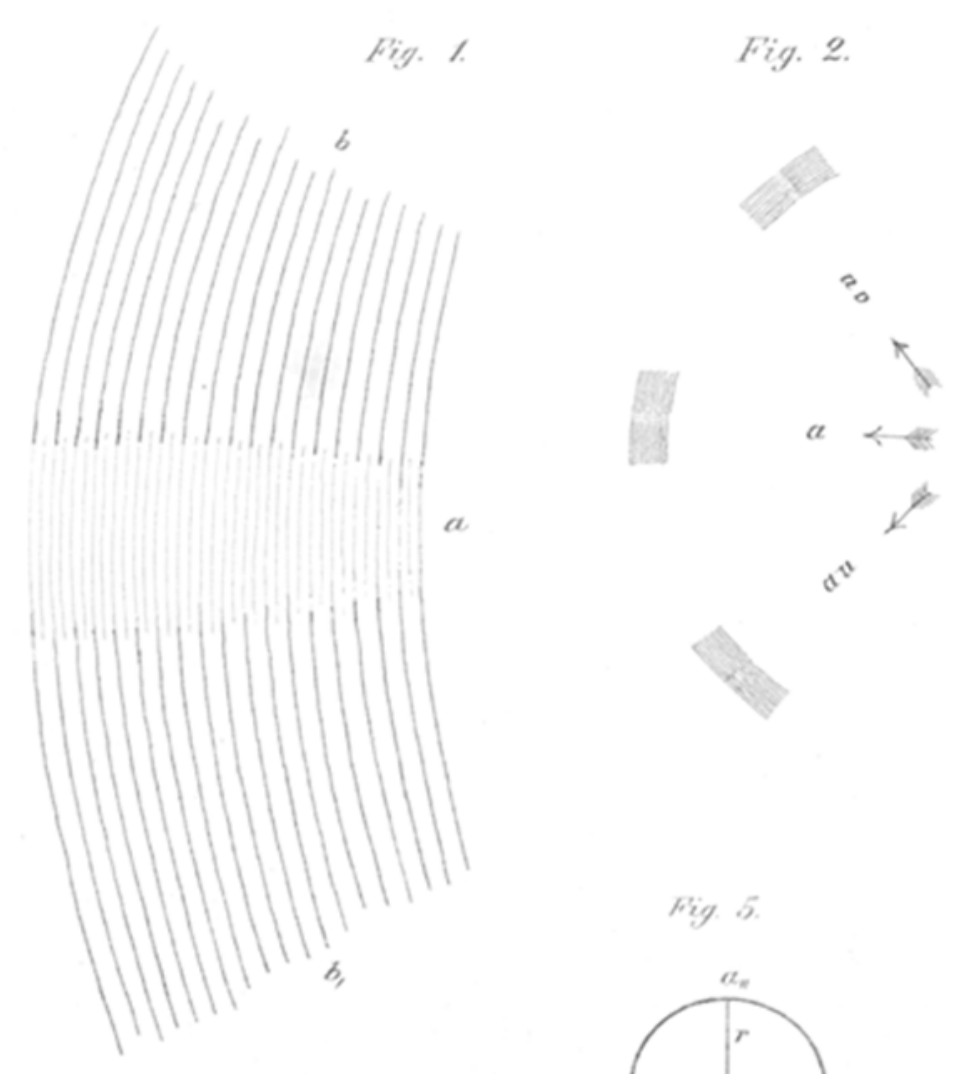

Fig. "

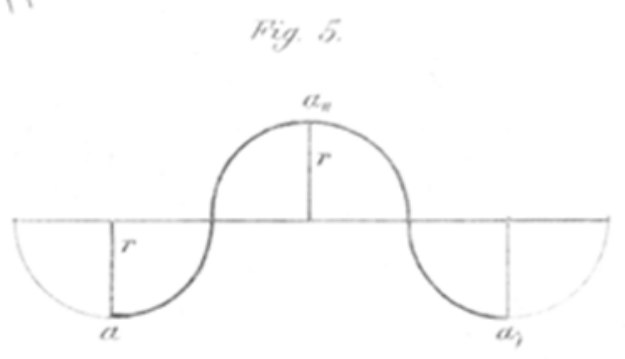

Fig. 3.

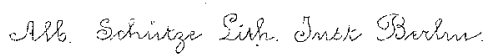

